\section{B-cell acute lymphoblastic leukemia with $t(4 ; 11)$ (q21;q23) in a young woman: evolution into mixed phenotype acute leukemia with additional chromosomal aberrations in the course of therapy}

\author{
Giovanni Carulli, ${ }^{1}$ Alessandra Marini, ${ }^{2}$ \\ Maria I. Ferreri, ${ }^{3}$ Antonio Azzarà, ${ }^{1}$ \\ Virginia Ottaviano, ${ }^{1}$ Tiziana Lari, ${ }^{2}$ \\ Melania Rocco, ${ }^{1}$ Stefano Giuntini, ${ }^{1}$ \\ Mario Petrini ${ }^{1}$ \\ ${ }^{1}$ Division of Hematology, Department \\ of Clinical and Experimental Medicine, \\ University of Pisa; ${ }^{2}$ Laboratory of Clinical \\ Pathology, Versilia Hospital, Lido di \\ Camaiore; ${ }^{3}$ Laboratory of Cytogenetics, \\ AOUP, Pisa, Italy
}

\section{Abstract}

About 5\% of adult B-cell acute lymphoblastic leukemias (B-ALL) are characterized by $\mathrm{t}(4 ; 11)(\mathrm{q} 21 ; \mathrm{q} 23)$, which confers peculiar features to this B-ALL subtype, including a very immature immunophenotype and poor prognosis. We describe the case of a 21-year-old female who presented with B-ALL carrying the $\mathrm{t}(4 ; 11)(\mathrm{q} 21 ; \mathrm{q} 23)$ and blasts positive for CD19, TdT, CD79a, CD38, HLA-DR. Before completing the Hyper-CVAD (hyperfractionated cyclophosphamide, vincristine, doxorubicin, and dexamethasone) therapy regimen, the B-cell leukemic clone still was detected, but an additional leukemic clone appeared, with morphology and immunophenotype (CD13, CD33, CD64, CD38, CD56, CD15, CD4 ${ }^{\text {dim }) ~ c o m p a t i b l e ~ w i t h ~ d e r i v a-~}$ tion from the myeloid/monocytic lineage. Karyotype showed the co-existence of three cell lines, with persistence of $t(4 ; 11)(q 21 ; q 23)$ and appearance of $+8,+12,+13$ and two $\operatorname{der}(4)$. The patient died because of disseminated intravascular coagulation. Our report describes a rare, possible evolution of such a subtype of B-ALL, with transformation into mixed phenotype acute leukemia in the course of therapy. This finding suggests a blast cell derivation from a common lymphoid/monocytic precursor leading to a final bilineal acute leukemia.

\section{Introduction}

Human acute lymphoblastic leukemia of the B lineage (B-ALL) involves clonal expansion of neoplastic $B$ precursors at one of the stages of
B-cell development. ${ }^{1}$ Some cases show rearrangements of the mixed-lineage leukemia $(M L L)$ gene, located at 11q23. MLL is able to recognize at least 64 partner genes, giving rise to at least 104 different $M L L$ rearrangements. ${ }^{2}$ For this reason, the most recent World Health Organization (WHO) classification of hematological myeloid neoplasms and acute leukemias identifies one sub-type of B-ALL [termed as B lymphoblastic leukemia/ lymphoma with $\mathrm{t}(\mathrm{v} ; 11 \mathrm{q} 23)$; $M L L$ rearranged] ${ }^{3}$ which involves all translocations of $M L L$ with one of the possible gene partners. About one third of B-ALL cases are characterized by $\mathrm{t}(4 ; 11)(\mathrm{q} 21 ; \mathrm{q} 23)$, which produces the $A F 4 / M L L$ fusion gene. ${ }^{4}$ This subtype of B-ALL accounts for about $5 \%$ of adult B-ALL, being more frequent in the pro-B-ALL subtypes. ${ }^{4,5}$

A very recent review shows that this subtype of B-ALL, ${ }^{6}$ although rare because of the low incidence of B-ALL in adults, is of great clinical interest due to biological, phenotypic and clinical features.

In the present paper we describe a case of very immature B-ALL with $\mathrm{t}(4 ; 11)(\mathrm{q} 21 ; \mathrm{q} 23)$ in a young woman. The disease was characterized by an uncommon evolution, since an additional leukemic clone, with myeloid/monocytoid phenotypic features, appeared in the course of induction therapy, probably accelerating the fatal outcome of the patient. Cytogenetics and in situ fluorescent hybridization (FISH) showed the co-existence of $\mathrm{t}(4 ; 11)(\mathrm{q} 21 ; \mathrm{q} 23)$ with a complex karyotype, which was characterized by three trisomies and the presence of two derivatives of chromosome. ${ }^{4}$

Therefore, the initial B-ALL with $\mathrm{t}(11 ; 14)$ (q21;q23) showed evolution into a bilineal acute leukemia (lymphoid and myeloid) compatible with the 2008 WHO entity defined as mixed phenotype acute leukemia (MPAL) with $\mathrm{t}(\mathrm{v} ; 11 \mathrm{q} 23), M L L$ rearranged. ${ }^{3}$ To the best of our knowledge, a similar evolution of B-ALL with $\mathrm{t}(11 ; 14)(\mathrm{q} 21 ; \mathrm{q} 23)$ has not been described so far.

\section{Case Report}

A Caucasian 21-year-old female presented at the Division of Hematology of the University of Pisa, Italy, with fever and anemia-related symptoms. A complete blood count showed hyperleukocytosis [white blood cell (WBC) 400 x $\left.10^{9} / \mathrm{L}\right]$, anemia and thrombocytopenia $(9 \mathrm{~g} / \mathrm{dL}$ and $50 \times 10^{9} / \mathrm{L}$, respectively). Her clinical history was silent, but she declared intake of heroin and cocaine. Physical examination showed mild splenomegaly. Whole body computed tomography confirmed the spleen enlargement (15 cm longitudinal diameter) and did not show pathologic lymph nodes.

Manual WBC differential count of peripher-
Correspondence: Giovanni Carulli, Division of Hematology, Santa Chiara Hospital, Via Roma 67. 56126 Pisa, Italy.

Tel. + 39.050.992731 - Fax: +39.050 .993378 .

E-mail: giovannicarulli@alice.it

Key words: acute lymphoblastic leukemia, acute myeloid leukemia, mixed phenotype acute leukemia, $M L L$ gene, $\mathrm{t}(4 ; 11)(\mathrm{q} 21 ; \mathrm{q} 23)$.

Contributions: GC evaluated the patient and wrote the manuscript; AM, TL, VO and SG carried out flow cytometry assays; MIF performed cytogenetics and FISH; AA performed morphologic analysis; MR and MP were responsible for patient's management.

Conflict of interests: the authors report no potential conflict of interests.

Received for publication: 6 March 2012.

Revision received: 19 April 2012.

Accepted for publication: 20 May 2012.

This work is licensed under a Creative Commons Attribution NonCommercial 3.0 License (CC BYNC 3.0).

(C) Copyright G. Carulli et al., 2012

Licensee PAGEPress, Italy

Hematology Reports 2012; 4:e15

doi:10.4081/hr.2012.e15

al blood showed $90 \%$ blasts without morphologic differentiation (Figure 1A), 2\% neutrophils, $8 \%$ small lymphocytes. Blasts resulted negative for myeloperoxidase stain. Flow cytometric analysis was therefore performed using a wide monoclonal antibody panel and a six-color method: blasts were positive for CD19, TdT, CD79a, CD38, CD58, HLA-DR (Figure 2A-F).

Bone marrow samples obtained from an aspirate were processed for light microscope evaluation, immunophenotyping, cytogenetics, polymerase chain reaction (PCR) assays for $\mathrm{IgH}$ gene rearrangement.

A massive infiltration by blasts with apparent lymphoid morphology was detected and immunophenotyping confirmed the results obtained with peripheral blood evaluation.

To perform PCR assays for $\mathrm{IgH}$ gene clonality assessment, mononuclear cells were separated by Ficoll/Hypaque gradient from bone marrow, and suitable aliquots were utilized for PCR tests after spectrophotometric quantitative evaluation. Fluorescent PCR reactions for IgH clonality evaluation were carried out with CDR3-specific consensus primer and analyzed by ABI PRISM 3100 (Applied Biosystems). ${ }^{7}$ PCR tests showed a clonal $I g H$ gene rearrangement. Finally cytogenetics, performed by using the Q-banding technique, revealed a karyotype with $\mathrm{t}(4 ; 11)(\mathrm{q} 21 ; \mathrm{q} 23)$ in $90 \%$ of metaphases.

The final diagnosis B-ALL with $t(4 ; 11)$ 
(q21;q23) was made. Chemotherapy with the dose-intensive phase of hyperfractionated cyclophosphamide, vincristine, doxorubicin, and dexamethasone (Hyper-CVAD) regimen was therefore given. Therapy included 8 cycles of dose-intensive therapy courses of HyperCVAD (courses 1, 3, 5, and 7) alternating with high-dose methotrexate and cytosine arabinoside (courses 2, 4, 6, and 8). Intrathecal central nervous system prophylaxis was also given with methotrexate or cytosine arabinoside, and recombinant human granulocyte colonystimulating factor was administered as a supportive care. ${ }^{8}$

After six of the established eight courses of therapy, although complete clearance of blasts from peripheral blood was obtained, minimal residual disease $(0.2 \%$ by flow cytometry), was detected in the bone marrow, along with the presence of a small leukemic clone in cerebrospinal fluid samples. The final two cycles of therapy were programmed, but the patient refused further antiblastic therapies and was dismissed from our hospital.

At home, her clinical conditions showed a rapid worsening and two weeks later she was admitted at Versilia Hospital, Italy. The patient presented with hyperleukocytosis $\left(220 \times 10^{9} / \mathrm{L}\right)$, hemoglobin $7 \mathrm{~g} / \mathrm{dL}$, platelets $10 \times 10^{9} / \mathrm{L}$, cutaneous hemorrhages (petechiae, purpura), laboratory findings of disseminated intravascular coagulation (low fibrinogen, prolonged prothrombin time and a-PTT, high D-dimer levels, low antithrombin levels), fever.

The observation of peripheral blood smears showed blasts $95 \%$, which consisted of two different clones, the former being represented by blast cells with lymphoid appearance (about $10 \%$ ), the latter being represented by cells with a larger size, abundant cytoplasm, giant nucleus with irregular profile (about 90\%) (Figures $1 \mathrm{~B}$ and $1 \mathrm{C}$ ). Some cells were classified as atypical monocytoid cells (Figure 1C) and very few blasts showed cytoplasmic granulations (Figure 1B). Immunophenotyping of circulating blasts showed a peculiar CD45/SSC dotplot, with the presence of two distinct blast cell populations. Blasts with small forward scatter (FSC) and side scatter (SSC), which accounted for $10 \%$, showed the phenotypic characteristics observed at diagnosis and were classified as belonging to the B-cell lineage. The majority of blasts (90\%) were characterized by larger FSC and SSC and appeared to derive from a myeloid/monocytic clone, being positive for CD13, CD33, CD64, CD15, CD56 and CD4 ${ }^{\mathrm{dim}}$ (Figure 3).

A new evaluation of karyotype confirmed the presence of the sole abnormality $\mathrm{t}(4 ; 11)$ $(q 21 ; q 23)$ in $10 \%$ of metaphases, and showed the appearance of two additional cell lines: one with 50 chromosomes, $\mathrm{t}(4 ; 11)(\mathrm{q} 21 ; \mathrm{q} 23)$, trisomy of chromosomes number 8,12 and 13 , and a not well defined supernumerary marker
(Figure 4A); the other one with 50 chromosomes, $\mathrm{t}(4 ; 11)(\mathrm{q} 21 ; \mathrm{q} 23)$, trisomy of chromosomes number 8,12 and 13 , and a derivative of chromosome 4 [der(4)] (Figure 4B). This latter anomaly was confirmed by FISH, which was performed on metaphases using whole chromosome painting n. 4 and n. 11 probes (Cytocell Inc. UK) and detected two der(4) (Figure 5). It was not possible to perform other studies, with the exception of a new PCR for IgH rearrange- ment, which showed persistence of a clonal pattern. The patient died because of disseminated intravascular coagulation.

\section{Discussion}

The B-ALL subtype carrying $\mathrm{t}(4 ; 11)(\mathrm{q} 21 ; \mathrm{q} 23)$ is a rare event, representing $5-10 \%$ of adult
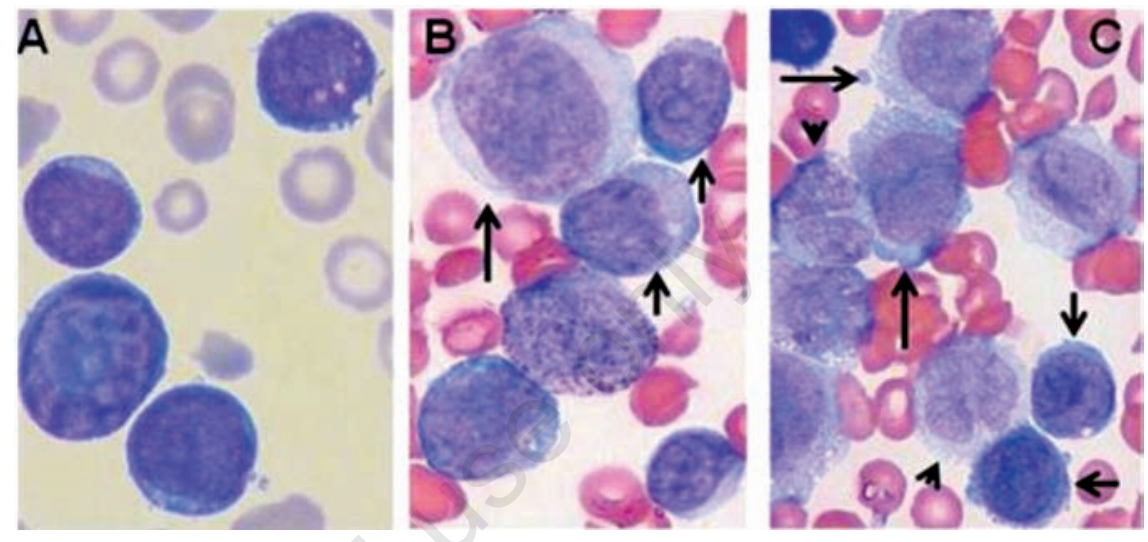

Figure 1. Morphology of blast cells at diagnosis (A) and in the course of fatal evolution $(B, C)$. A) blasts appear with lymphoid morphology; B,C) blasts consist of two different clones. Blasts with lymphoid morphology (arrows) show smaller size. The additional blast cell population consists of cells of larger size and more abundant cytoplasm (long arrows). Some monocytoid cells (arrowheads) and one myeloblast with evident cytoplasmic granulations (B) are also shown. Peripheral blood, May-Grünwald-Giemsa staining (1000x).
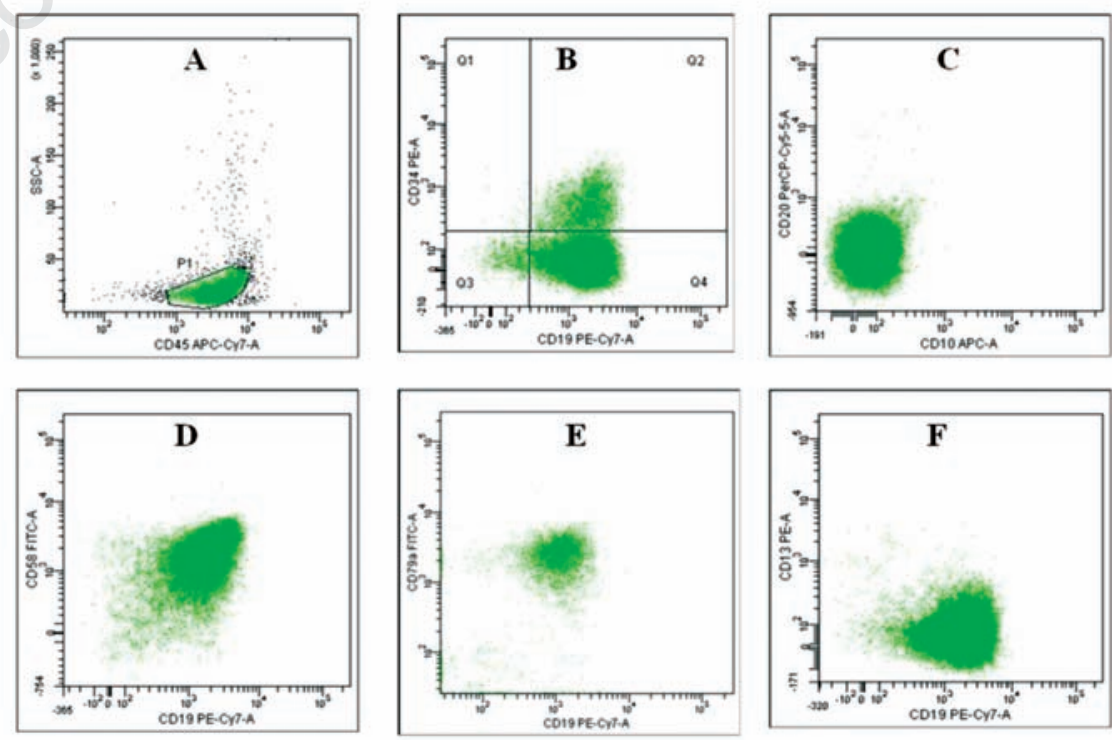

Figure 2. Immunophenotype of blasts at diagnosis in peripheral blood samples. A) CD 45/SSC dot-plot. Blasts are included in P1 population. B) blasts are CD19-positive, with a minority of them $(18 \%)$ being $C D 34-$ positive $(\mathrm{Q} 2$ quadrant). Blasts are CD10and CD20-negative (C) and CD58-positive (D). E) positivity of CD79a. F) blasts are negative for CD13. 
cases of B-ALL which, in turn, is a disease with very low incidence (less than 1 per 100,000 persons/year). In an ample study published by the Medical Research Council and the Eastern Cooperative Oncology Group, which involved 1522 patients with acute lymphoblastic leukemia, only 54 showed a B-ALL with $\mathrm{t}(4 ; 11)(q 21 ; q 23) .{ }^{9}$ This type of B-ALL shows peculiar features. From a clinical point of view, it is characterized by elevated WBC counts, high incidence of central nervous system involvement, frequent hepato-splenomegaly, poor clinical outcome both in children and in adults. ${ }^{6}$ Due to these reasons, B-ALL with $\mathrm{t}(4 ; 11)$ are considered as high risk leukemias. ${ }^{5}$ The presence of $\mathrm{t}(4 ; 11)(q 21 . q 23)$ is more frequent in B-ALL deriving from a very immature B-cell precursor. ${ }^{5}$ This feature is suggested by the phenotype displayed by blast cells, which are generally positive for CD19 and markers associated with an immature immunophenotype, as demonstrated by the co-expression of CD19, TdT, CD79a and often CD34, and the absence of CD10 and CD20. Negativity for CD10 is always observed. ${ }^{10}$ Because of this peculiar immunophenotype, the B-ALL subtype carrying $\mathrm{t}(4 ; 11)(\mathrm{q} 21 ; \mathrm{q} 23)$ represents about $40 \%$ of all forms of pro-B ALL in adults. Several but not all cases show co-expression of some myeloid markers, such as CD15 and CD65, with constant negativity of CD13 and CD33. ${ }^{4}$

In our case findings of typical B-ALL with $\mathrm{t}(4 ; 11)(\mathrm{q} 21 ; \mathrm{q} 23)$ were found at diagnosis. Central nervous system involvement was detected during the course of disease, in a phase of bone marrow minimal residual disease.

The poor outcome of our patient was favored by an unexpected event, represented by the appearance of an additional leukemic clone with both morphological and immunophenotypical properties which could be attributed to the myeloid/monocytic lineage. In fact, during the terminal and fatal phase of disease, two distinct blast cell populations were found, the former with a B-cell phenotype and the latter with a separate phenotype characterized by coexpression of markers frequently found in acute myeloid leukemias with prevalent monocytic differentiation, ${ }^{11,12}$ such as CD13, CD33, CD64, CD15, CD4. Co-expression of B-cell markers and myeloid markers was not detected. The appearance of this additional leukemic clone was accompanied by the preponderant presence of additional cytogenetic aberrations, such as trisomy of chromosomes 8, 12 and 13 . Trisomy 8 is a relatively frequent $(10-15 \%$ of cases) abnormality in acute myeloblastic leukemias (AML), in which is associated with a poor prognosis. Isolated trisomy 12 is an infrequent finding both in AML and in B-ALL and has been described in highly undifferentiated acute leukemias. Isolated trisomy 13 is another marker with a negative impact on prognosis and seems to be associated with the
FAB M0 subtype and very low remission rates. Thus, the association of these three cytogenetic anomalies should be interpreted as biologic marker with an additional negative impact on disease. The presence of double der(4) [derivative of $t(4 ; 11)(q 21 ; q 23)]$ is likely to have given a synergistic effect to the leukemic phenotype as well as an attractive leukemic subtype with poor prognosis. The latter finding is consistent with the initial lymphoblastic clone, since a der(4) chromosome can been found in up to $65 \%$ of patients with $\mathrm{t}(4 ; 11)(\mathrm{q} 21 ; \mathrm{q} 23)$. $^{13}$

There is evidence of an interesting association of $M L L$ gene rearrangements and MPAL. The 2008 WHO classification has identified a particular subtype of MPAL termed mixed phenotype acute leukemia with $t(v ; 11 q 23) ; M L L$ rearranged ${ }^{3}$ which can show either the presence of blasts with simultaneous expression of markers of different lineages or the presence of two populations of blasts with distinct phenotype. In a recent analysis of 100 cases of MPAL, Matutes et al. ${ }^{14}$ found only three cases with $M L L$ gene rearrangement due to $\mathrm{t}(4 ; 11)(\mathrm{q} 21 ; \mathrm{q} 23)$ at initial presentation. In a less recent report Johansson et al. ${ }^{15}$ analyzed 183 cases of $\mathrm{t}(4 ; 11)(\mathrm{q} 21 ; \mathrm{q} 23)$ and found that $34 \%$ were children, $95 \%$ were B-ALL and that only one case was a biphenotypic acute leukemia. Rubnitz et al. ${ }^{16}$ reported a series of 35 children with MPAL and found four cases with $M L L$ gene rearrangement: only two cases showed a bilineal pattern (myeloid and lymphoid B), but $\mathrm{t}(4 ; 11)(\mathrm{q} 21 ; \mathrm{q} 23)$ was not detect-
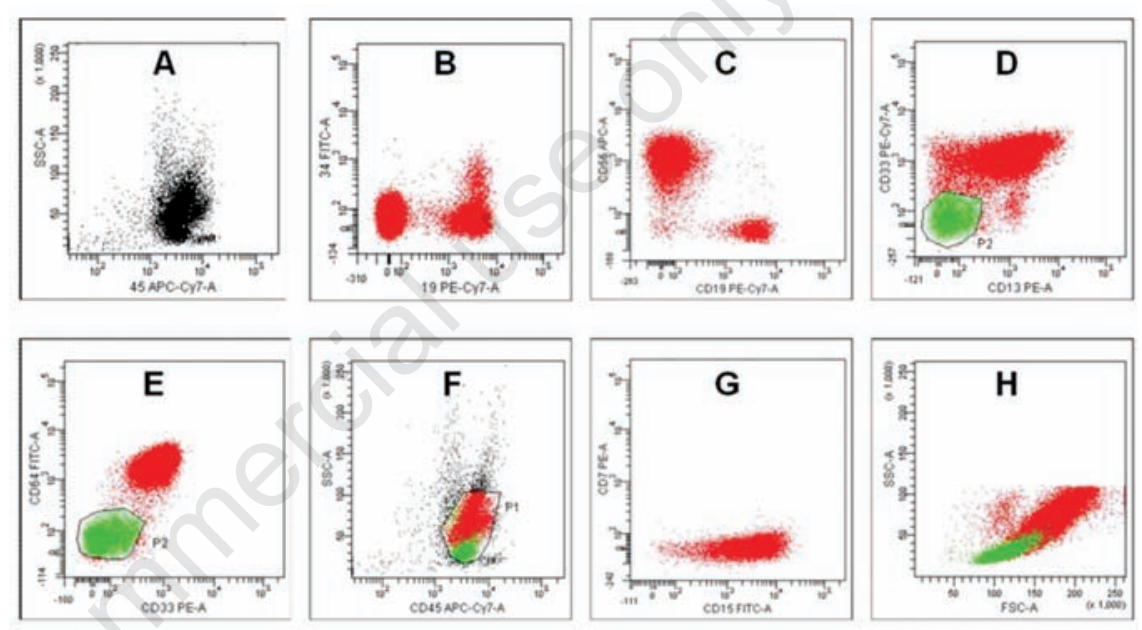

Figure 3. Immunophenotype of peripheral blood blasts during the terminal phase of disease. A) CD45/SSC dot-plot. B-E) Pattern of expression of myeloid markers (CD13,CD33,CD64), of CD34, CD19 and CD56. F) Different SSC properties of lymphoid and myeloid blasts. G) CD15 positivity pattern of myeloid blasts. H) Different FSC and SSC properties of lymphoid and myeloid blasts. P1: global blast population. Green dots and P2: lymphoid (CD19-positive) blasts.
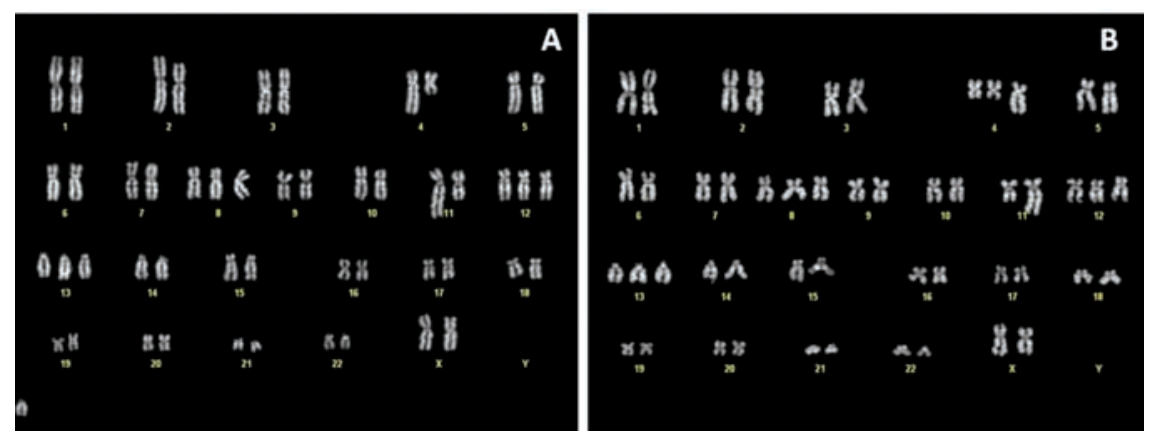

Figure 4. Representative Q-banded karyotype of the two additional leukemic clones. A) $50, \mathrm{XX}, \mathrm{t}(4 ; 11)(\mathrm{q} 21 ; \mathrm{q} 23),+8,+12,+13,+\mathrm{m} . \mathrm{B}) 50, \mathrm{XX}, \mathrm{t}(4 ; 11)(\mathrm{q} 21 ; \mathrm{q} 23),+8,+12,+13$, $+\operatorname{der}(4) t(4 ; 11)(q 21 ; q 23)$. 
ed. Sporadic additional cases of acute biphenotypic leukemia associated with complex $M L L$ gene rearrangement have been reported. ${ }^{17,18}$

Thus, although $M L L$ gene rearrangement with $\mathrm{t}(4 ; 11)(\mathrm{q} 21 ; \mathrm{q} 23)$ can be observed in cases of MPAL, this peculiar subtype of acute leukemia seems to be very rare at first presentation, probably being more frequent in children.

Another possible presentation of cases of BALL with $\mathrm{t}(4 ; 11)(\mathrm{q} 21 ; \mathrm{q} 23)$ is represented by phenotypic shift. Indeed, phenotypic changes of blast cells in this sub-type of B-ALL have rarely been reported. Lineage switch from lymphoid to myeloid phenotype was reported by Trikalinos et al. ${ }^{19}$ in a young woman after allogeneic stem cell transplantation. Germano et $a l .{ }^{20}$ reported a pediatric case with two consecutive phenotypic changes from lymphoid to myeloid lineage and vice versa. The phenotypic switches were observed after lineage-targeted chemotherapy in both instances. Two additional pediatric cases of switch from acute lymphoblastic leukemia to acute myeloid leukemia, involving $M L L$ gene rearrangements, were reported by Jiang et al. ${ }^{21}$ and by Stasik et al. ${ }^{22}$

In all the above cases, it could be thought that the lineage switch might represent the expansion of a pre-existing minor population of blasts of myeloid lineage following chemotherapy-induced suppression of the initial major lymphoid blast population. Another possible interpretation, according to a current opinion about the existence of a common lymphoid-myeloid precursor, ${ }^{23}$ is that leukemic clones carrying rearrangements of the $M L L$ gene are able to differentiate along the lymphoid or myeloid lineage depending on activation/deactivation of differentiation programs, and that chemotherapy may play a role in this scenario.

The pathogenetic interpretation of our case

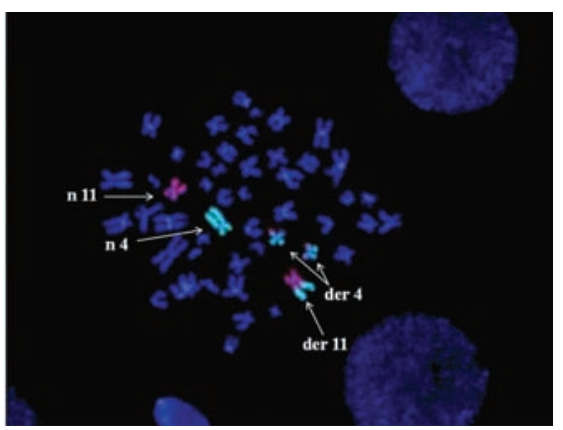

Figure 5. Fluorescent hybridization analysis performed on metaphases using whole chromosome painting n. 4 and n.11 probes. Two derivative chromosomes 4 were found. may be facilitated by previous reports showing a relationship between the $\mathrm{t}(4 ; 11)$ translocation and a singular association of lymphoid and monocytic lineage in human leukemic cells lines. Stong et al. ${ }^{24}$ reported a study concerning a cell line derived from the bone marrow of a female adult patient in first relapse. The cell line, termed RS4;11, showed lymphoid morphology, various B-associated markers, HLA-DR expression, clonal rearrangement of heavy chain and light chain genes of immunoglobulins, and lacked the CALLA antigen (corresponding to CD10). Such cells acquired cytochemical and ultrastructural features following treatment with the phorbol ester TPA. Similar results had been observed in two pediatric cases. ${ }^{25}$ In a more recent report, Dunphy et al. ${ }^{26}$ described a case of a CD15-positive, $\mathrm{t}(4 ; 11)$-positive B-ALL which relapsed as acute leukemia with monocytoid features after allogeneic bone marrow transplant. The initial CD19-positive clone, however, was no longer present, although persistence of clonal rearrangement of the $I g H$ gene could be detected.

The very few previous reports are consistent with a possible relationship between the B-lineage and the monocytic lineage in cases of acute leukemia with $\mathrm{t}(4 ; 11)(\mathrm{q} 21 ; \mathrm{q} 23)$ and suggest the possible involvement of a common lymphoid-myeloid precursor which, sometimes, might show a predominant monocytic differentiation program.

Indeed, Zangrando et al., ${ }^{27}$ employing gene expression profiling assays, found that, in cases of acute leukemia with $M L L$ gene rearrangements, a subset of genes identifies $M L L$-specific rearrangements and is able to distinguish ALL from AML. In addition, a small subset of genes (MEIS1, ZEB2, SRGAP2P1, TMEM30A, AK2, TMED2, HIPK3 and FAM62B) showed marked up-regulation in patients with MLL mutation, both in ALL and AML. These findings appear of particular interest, because seem to emphasize the role of $M L L$ gene in the onset of cases of BPAL.

\section{Conclusions}

Because of the above considerations, it appears that in our patient the initial B-ALL with $t(4 ; 11)(q 21 ; q 23)$ showed, in the course of therapy, an evolution into a MPAL with the same $M L L$ gene rearrangement, but with additional chromosomal aberrations probably conferred by the additional, non-lymphoblastic, leukemic clone.

We think that the case described in the current report adds novel information about the possible evolution of B-ALL with $\mathrm{t}(4 ; 11)$ $(q 21 ; 23)$. Not only phenotypic switches are possible after chemotherapy, but additional leukemic clones, with myeloid/monocytoid properties, may arise in the course of antiblastic therapy, probably deriving from a common precursor. Thus, a wide panel of MoAb should be used when monitoring B-ALL positive for $\mathrm{t}(4 ; 11)(\mathrm{q} 21 ; \mathrm{q} 23)$, in order to detect eventual phenotypic shifts and/or appearance of additional leukemic clones. Due to the poor outcome which characterizes both B-ALL with $t(4 ; 11)(q 21 ; q 23)$ and BPAL, early detection of such uncommon evolution of this B-ALL subtype may have practical implications in terms of follow-up strategies.

\section{References}

1. Faderl S, 0'Brien S, Pui CH, et al. Adult acute lymphoblastic leukemia. Cancer 2010;116:1165-76.

2. Meyer C, Kowarz E, Hofmann J, et al. New insights to the MLL recombinome of acute leukemias. Leukemia 2009;23:1404-9.

3. Vardiman JW, Thiele J, Arber DA, et al. The 2008 revision of the World Health Organization (WHO) classification of myeloid neoplasms and acute leukemia: rationale and important changes. Blood 2009;114:937-51.

4. Mancini M, Scappaticci D, Cimino G, et al. A comprehensive genetic classification of adult acute lymphoblastic leukemia (ALL): analysis of the GIMEMA 0496 protocol. Blood 2005;105:3430-41.

5. Gleissner B, Goekbuget N, Rieder H, et al. CD10- pre-B acute lymphoblastic leukemia (ALL) is a distinct high-risk subgroup of adult ALL associated with a high frequency of MLL aberrations: results of the German Multicenter Trials for Adult ALL (GMALL). Blood 2005;106:4054-56.

6. Marchesi F, Giradi K, Avvisati G. Pathogenetic, clinical, and prognostic features of adult $t(4 ; 11)(q 21 ; q 23) / M L L-A F 4$ positive B-cell acute lymphoblastic leukemia. Adv Hematol 2011;2011:621-7.

7. Galimberti S, Brizzi F, Mameli M, Petrini M. An advantageous method to evaluate IgH rearrangement and its role in minimal residual disease detection. Leuk Res 1999;23:921-9.

8. Kantarjian H, Thomas DA, O'Brien S, et al. Long-term follow-up results of hyperfractionated cyclophosphamide, vincristine, doxorubicin, and dexamethasone (HyperCVAD), a dose-intensive regimen, in adult acute lymphocytic leukemia. Cancer 2004;15:2778-801.

9. Moorman AV, Harrison CJ, Buck GA, et al. Karyotype is an independent prognostic factor in adult acute lymphoblastic leukemia (ALL): analysis of cytogenetic data from patients treated on the Medical 
Research Council (MRC) UKALLXII/ Eastern Cooperative Oncology Group (ECOG) 2993 trial. Blood 2007;109:318997.

10. Attarbaschi A, Mann G, König M, et al. Mixed lineage leukemia-rearranged childhood pro-B and CD10-negative pre-B acute lymphoblastic leukemia constitute a distinct clinical entity. Clin Cancer Res 2006;12:2988-94.

11. Dunphy CH, Orton SO, Mantell J. Relative contributions of enzyme cytochemistry and flow cytometry to the evaluation of acute myeloid leukemias with a monocytic component and of flow cytometric immunophenotyping to the evaluation of absolute monocytoses. Am J Clin Pathol 2004;122:865-74.

12. Vidriales MB, Orfao A, López-Berges MC, et al. Light scatter characteristics of blast cells in acute myeloid leukaemia: association with morphology and immunophenotype. J Clin Pathol 1995;48:456-62.

13. Janssen JW, Ludwig WD, Borkhardt A, et al. Pre-pre-B acute lymphoblastic leukemia: high frequency of alternatively spliced ALL1-AF4 transcripts and absence of minimal residual disease during complete remission. Blood 1994;84:3835-42.

14. Matutes E, Pickl WF, Van't Veer M, et al. Mixed-phenotype acute leukemia: clinical and laboratory features and outcome in 100 patients defined according to the WHO
2008 classification. Blood 2011;117:316371.

15. Johansson B, Moorman AV, Haas OA, et al. Hematologic malignancies with $\mathrm{t}(4 ; 11)$ (q21;q23). A cytogenetic, morphologic, immunophenotypic and clinical study of 183 cases. Leukemia 1998;12:779-87.

16. Rubnitz JE, Onciu M, Pounds S, et al. Acute mixed lineage leukemia in children: the experience of St Jude Children's Research Hospital. Blood 2009;113:5083-9.

17. Derwich K, Sedek L, Meyer C, et al. Infant acute bilineal leukemia. Leuk Res 2009;33:1005-8.

18. Weir EG, Ali Ansari-Lari M, Batista DA, et al. Acute bilineal leukemia: a rare disease with poor outcome. Leukemia 2007;21: 2264-70.

19. Trikalinos NA, Soupir CP, Dey BR. Lineage switch of acute lymphocyctic leukaemia with $\mathrm{t}(4 ; 11)(\mathrm{q} 21 ; \mathrm{q} 23)$ into acute myeloid leukaemia in an adult patient after allogeneic stem cell transplantation. Br J Haematol 2009;145:262-4.

20. Germano G, Pigazzi M, Del Giudice L, et al. Two consecutive immunophenotypic switches in a child with MLL-rearranged acute lymphoblastic leukemia. Haematologica 2006;91:29-31.

21. Jiang G, Roman E, Nandula SV, et al. Congenital MLL-positive B-cell acute lymphoblastic leukaemia (B-ALL) switched lineage at relapse to acute myelocytic leukaemia (AML) with persistent $\mathrm{t}(4 ; 11)$ and $\mathrm{t}(1 ; 6)$ translocations and $\mathrm{JH}$ gene rearrangement. Leuk Lymphoma 2005; 46 : 1223-7.

22. Stasik C, Ganguly S, Cunningham MT, et al. Infant acute lymphoblastic leukaemia with $\mathrm{t}(11 ; 16)(\mathrm{q} 23 ; \mathrm{p} 13.3)$ and lineage switch into acute monoblastic leukaemia. Cancer Genet Cytogenet 2006;168:146-9.

23. Kawamoto H, Wada H, Katsura Y. A revised scheme for developmental pathways of hematopoietic cells: the myeloid-based model. Int Immunol 2010;22:65-70.

24. Stong RC, Korsmeyer SJ, Parkin JL, et al. Human acute cell line with the $t(4 ; 11)$ chromosomal rearrangement exhibits $B$ lineage and monocytic characteristics. Blood 1985;65:21-31.

25. Nagasaka M, Maeda $\mathrm{S}$, Maeda $\mathrm{H}$, et al. Four cases of $\mathrm{t}(4 ; 11)$ acute leukemia and its myelomonocyic nature in infants. Blood 1983;61:1174-81.

26. Dunphy CH, Gardner LJ, Evans HL, Javadi N. CD15+ acute lymphoblastic leukemia and subsequent monoblastic leukemia. Arch Pathol Lab Med 2001;125:1227-30.

27. Zangrando A, Campo Dell'Orto M, Te Kronnie G, Basso G. MLL rearrangements in pediatric acute lymphoblastic and myeloblastic leukemias: MLL specific and lineage specific signatures. BMC Med Genomics 2009;2:36. 